\title{
Combined non-alcoholic fatty liver disease and type 2 diabetes in severely obese patients-medium term effects of sleeve gastrectomy versus Roux-en-Y-gastric bypass on disease markers
}

\author{
Franck Billmann ${ }^{1 \#}$, Sherehan El Shishtawi ${ }^{1 \#}$, Tom Bruckner ${ }^{2}$, Mostafa ElSheikh $^{3}$, \\ Beat Peter Müller-Stich ${ }^{1}$, Adrian Billeter ${ }^{1}$
}

${ }^{1}$ Department of Surgery, University Hospital of Heidelberg, Heidelberg, Germany; ${ }^{2}$ Institut für Medizinische Biometrie und Informatik, Universität Heidelberg, Heidelberg, Germany; ${ }^{3}$ Department of General Surgery, El-Gharbia Govenorate, Tanta, El gash St. Medical Campus, The Faculty of Medicine, Tanta, Egypt

Contributions: (I) Conception and design: F Billmann, S El Shishtawi, BP Müller-Stich, A Billeter; (II) Administrative support: F Billmann, BP Müller-Stich, A Billeter; (III) Provision of study materials or patients: F Billmann, BP Müller-Stich, A Billeter; (IV) Collection and assembly of data: F Billmann, S El Shishtawi; (V) Data analysis and interpretation: F Billmann, S El Shishtawi, BP Müller-Stich, A Billeter; (VI) Manuscript writing: All authors; (VII) Final approval of manuscript: All authors.

"These authors contributed equally to this work and should be considered as co-first authors.

Correspondence to: Adrian Billeter, MD, PhD. Department of Surgery, University Hospital of Heidelberg, Im Neuenheimer Feld 420 , D-69120 Heidelberg, Germany. Email: Adrian.Billeter@med.uni-heidelberg.de.

Background: We aimed to evaluate the medium-term efficacy of sleeve gastrectomy (SG) vs. Roux-en-Y gastric bypass (RYGB) on remission of non-alcoholic fatty liver disease (NAFLD) in patients with type 2 diabetes mellitus (T2DM).

Methods: We identified severely obese patients [body mass index (BMI) $>35 \mathrm{~kg} / \mathrm{m}^{2}$ ] with NAFLD (as defined by the Longitudinal Assessment of Bariatric Surgery Study) and T2DM (as defined by the American Association of Clinical Endocrinologists and the American College of Endocrinology) who underwent SG or RYGB in a single university surgical centre. The cohorts were match-paired and data were analysed after at least 3 years of follow up. The key outcomes measured were: (I) the improvement of liver function tests and NAFLD markers; (II) glycemic control and insulin resistance.

Results: Ninety-six patients were investigated; 44 (45.8\%) were women. The mean pre-operative BMI was $45.2 \mathrm{~kg} / \mathrm{m}^{2}$ in the SG and $42.0 \mathrm{~kg} / \mathrm{m}^{2}$ in the RYGB group. SG and RYGB both significantly reduced serum liver enzyme concentrations. NAFLD markers resolved 2 years after SG in all patients. In contrast, only 78\% and $80 \%$ of patients achieved remission of NAFLD 2 and 3 years after RYBG respectively. Both procedures resulted in comparable rates of remission of T2DM.

Conclusions: Bariatric surgery with SG may be preferable to RYGB for obese patients with NAFLD and T2DM based on the rates of remission of markers of these co-morbidities. However, our results need to be confirmed in prospective trials. Understanding the metabolic effects of specific bariatric surgical procedures may facilitate the development of a personalised approach to weight-loss surgery.

Keywords: Bariatric surgery; morbid obesity complications; non-alcoholic fatty liver disease (NAFLD); type 2 diabetes mellitus (T2DM); gastric bypass; sleeve gastrectomy (SG); transaminases

Submitted Feb 15, 2021. Accepted for publication May 11, 2021.

doi: $10.21037 / \mathrm{hbsn}-21-71$

View this article at: https://dx.doi.org/10.21037/hbsn-21-71 


\section{Introduction}

Non-alcoholic fatty liver disease (NAFLD) is frequently associated with obesity and affects an estimated 25-30\% of the population worldwide (1). In fact, NAFLD has a prevalence of $40-70 \%$ in patients with diabetes (1). Moreover, type 2 diabetes mellitus (T2DM) and obesity often occur together as part of the metabolic syndrome. Therefore, the combination of NAFLD, T2DM and obesity represents a major public health challenge, particularly in the developed world (2).

NAFLD is part of the spectrum of liver diseases, which ranges from reversible steatosis and steatohepatitis (NASH) to irreversible cirrhosis and liver cancer (3). In fact, T2DM is one of the strongest risk factors for progression of NAFLD to NASH, cirrhosis and hepatocellular carcinoma (HCC). Recent studies have also drawn attention to the prevalence of NASH in patients with body mass indices in the normal range (lean NASH), which is also associated with the development of liver disease complications. Thus, there is a pressing clinical need to identify effective therapies for NAFLD in patients with metabolic co-morbidities.

At present, there is lack of a drug-based therapy which results in long-term weight reduction and improves and/ or prevents obesity-related co-morbidities, including NAFLD-related liver failure and HCC (4-6). In contrast, a bariatric surgery-based approach, particularly for patients with a body mass index $(\mathrm{BMI}) \geq 35 \mathrm{~kg} / \mathrm{m}^{2}$, may not only achieve the goal of weight reduction but may also ameliorate obesity-related co-morbidities. For example, bariatric surgery can improve glycaemic control in T2DM within days of surgery, even in patients with a BMI $<35 \mathrm{~kg} / \mathrm{m}^{2}(6-8)$. Bariatric surgery has also been shown to reduce cardiovascular- and overall mortality in obese patients with T2DM and can prevent the development of microand macrovascular complications (9). Whilst NAFLD is not currently a stand-alone indication for bariatric surgery (10), evidence is now emerging that bariatric surgery can modulate the clinical course of NAFLD and NASH (11). Although weight loss by itself can lead to an improvement or resolution in NAFLD (12), mechanisms independent of weight loss have also been suggested. Moreover, bariatric procedures not only decrease the likelihood of progression to hepatic decompensation but may also have the potential to reverse existing liver damage (13).

However, the term bariatric surgery encompasses many procedures and the extent to which the specific surgical approach, sleeve gastrectomy (SG) or Roux-en-Y gastric bypass (RYGB), influences the course of NAFLD and other obesity-related comorbidities is unclear. This uncertainty is at least partially due to the preponderance of short-term studies in the published literature, often with incomplete descriptions of the surgical approach and patients' comorbidities (14-17). Moreover, few studies have stratified patients on the basis of co-existing NAFLD and T2DM (15). Therefore, there is a need for medium- to long-term studies to specifically examine the metabolic effects of bariatric surgery in obese patients with NAFLD and T2DM, particularly given that these co-morbidities are associated with the highest risk of progression of NAFLD to cirrhosis and HCC. The aim of this matched-pair analysis was to compare the medium-term effects of SG vs. RYGB on remission of pre-existing NAFLD in patients with concomitant T2DM. We present the following article in accordance with the STROBE reporting checklist (available at https://hbsn.amegroups.com/article/view/10.21037/ hbsn-21-71/rc).

\section{Methods}

\section{Study design and setting}

The study was conducted in accordance with the Declaration of Helsinki (as revised in 2013). The study was approved by institutional review board (University of Heidelberg) and individual consent for this retrospective analysis was waived. It was a single-centre retrospective cohort study. All patients were operated upon by experienced surgeons in a certified centre for bariatric surgery. Pre-operative assessment was performed by a multi-disciplinary team consisting of bariatric surgeons, endocrinologists, dieticians, psychologists and psychiatrists, all with experience in evaluating patients before and after bariatric surgery. The operation technique was as previously described (15); all patients underwent laparoscopic procedures without use of a robotic assistance. Patient, procedural and follow-up data were collected between 2006 and 2019.

\section{Cohort and follow-up}

Patients included in this study were diagnosed with severe obesity $\left(\mathrm{BMI}>35 \mathrm{~kg} / \mathrm{m}^{2}\right)$, NAFLD and T2DM, as defined by the American Association of Clinical Endocrinologists and the American College of Endocrinology (18). Patients were treated according to the national guidelines for bariatric and metabolic surgery (19). All patients underwent 
an endocrinological assessment to exclude obesity secondary to a hormonal aetiology, as well as psychological evaluation to exclude any contra-indications to bariatric surgery. Furthermore, all patients received nutritional counselling by dieticians experienced in the care of bariatric patients. Prior to 2018, all patients underwent 6-month of conservative medical weight loss therapy. After 2018, patients with a BMI $\geq 50 \mathrm{~kg} / \mathrm{m}^{2}$ or a T2DM and a BMI $\geq 40 \mathrm{~kg} / \mathrm{m}^{2}$ underwent the bariatric-metabolic operation without 6 months of conservative medical weight loss therapy, as per national guidelines.

Follow-up for each patient began on the day of surgery. The choice of the procedure, SG or RYGB, was based on technical feasibility, which was determined after laparoscopic exploration. Patient preference was also taken into account. Patients were excluded if (I) they did not complete a standard surgical follow-up of at least 3 years, (II) they were younger than 18 years of age or were unable to give informed consent, (III) they were diagnosed preoperatively with a significant hiatal hernia or gastroesophageal reflux disease (GERD), (IV) there was inadequate assessment of NAFLD and glycemic control (i.e., complete follow-up values were not available).

\section{Outcomes}

Demographic, clinical, and peri-operative (including clinical, pathological, biochemical and co-morbidity) data were collected for all patients included in this study.

The investigated outcomes were: (I) the improvement of liver function tests and NAFLD [alanine transaminase (ALT), aspartate transaminase (AST), NAFLD Fibrosis Score, Hepatic Steatosis Index]; (II) glycemic control (fasting glucose, HbA1c, insulin use, metformin use), insulin resistance; (III) mortality and morbidity; and (IV) \% excess body weight loss (\% EBW) and \% total body weight loss (\% TWL).

\section{Definition of NAFLD}

NAFLD was defined based on an elevated level of ALT ( $>35 \mathrm{U} / \mathrm{L}$ ) according to previous studies (20). The cut-off of $35 \mathrm{U} / \mathrm{L}$ is higher than the one used by Prati et al. in their definition of healthy ranges of ALT $(<19$ U/L for women and $<30 \mathrm{U} / \mathrm{L}$ for men) (21). However, given that serum ALT alone is merely a screening tool, neither fully sensitive nor specific for the NAFLD, the NAFLD Fibrosis Score (NFS) was also investigated as in previous studies (22). This non-invasive score was proposed by Angulo et al. (23) as a reliable surrogate marker for the severity of NAFLD associated liver fibrosis. This score is based on the following formula: NFS $=-1.675+0.037 \times$ age (years) $+0.094 \times$ BMI $\left(\mathrm{kg} / \mathrm{m}^{2}\right)+1.13 \times \mathrm{IFG} /$ diabetes $($ yes $=1, \mathrm{no}=0)+0.99 \times \mathrm{AST} /$ ALT ratio $-0.013 \times$ platelet $\left(\times 10^{9} / \mathrm{L}\right)-0.66 \times$ albumin $(\mathrm{g} / \mathrm{L})$. NFS is valid as an index of fibrosis. In the absence of direct measurement of liver fat [e.g., biopsy, ultrasound or magnetic resonance imaging (MRI)], we used the Hepatic Steatosis Index (HSI). This score is based on the following formula: $\mathrm{HSI}=8 \times \mathrm{ALT} / \mathrm{AST}$ ratio $+\mathrm{BMI}+2$ (if diabetes) +2 (if female), with values $<30$ ruling out and values $\geq 36$ ruling in steatosis.

\section{Assessment of glycemic control}

Fasting glucose, HbA1c and insulin were determined in serum samples collected pre-operatively and during the follow-up. Laboratory parameters were measured by the certified central laboratory of our University Hospital as part of the regular clinical follow-up. Insulin resistance was assessed by the homeostasis model assessment for insulin resistance (HOMA-IR) according to Matthews et al. (24) using the following formula: [glucose $(\mathrm{mg} / \mathrm{dL}) \times$ insulin $(\mathrm{mU} / \mathrm{L})] / 405$. Remission of T2DM was achieved when all of the following criteria were fulfilled: fasting glucose $<100 \mathrm{mg} / \mathrm{dL}, \mathrm{HbA} 1 \mathrm{c}<6.0 \%$ and no use of antidiabetic medication, according to the 2009 consensus conference (25).

\section{Statistical analysis}

All analyzes were performed using the PRISM 8 GraphPad software (GraphPad Software, San Diego, USA). Continuous variables were summarized as mean and standard deviation (SD) and median (interquartile range, IQR). Categorcial variables were registered as $\mathrm{n}(\%)$. Statistical comparisons of quantitative variables were performed with Student's $t$ test or Mann-Whitney-U test. For categorical variables, the Pearson's Chi ${ }^{2}$ test or Fisher's exact test were used. All statistical tests were two-sided, and $\mathrm{P}$ values of less than 0.05 were considered statistically significant.

\section{Matching criteria}

For the purpose of comparing the treatment outcomes and morbidity between the groups (SG vs. RYGB) and in order to minimize selection bias, a matched-pair analysis was performed. The technique of bariatric procedure used (SG vs. RGYB) was entered in the matched-pair model as 


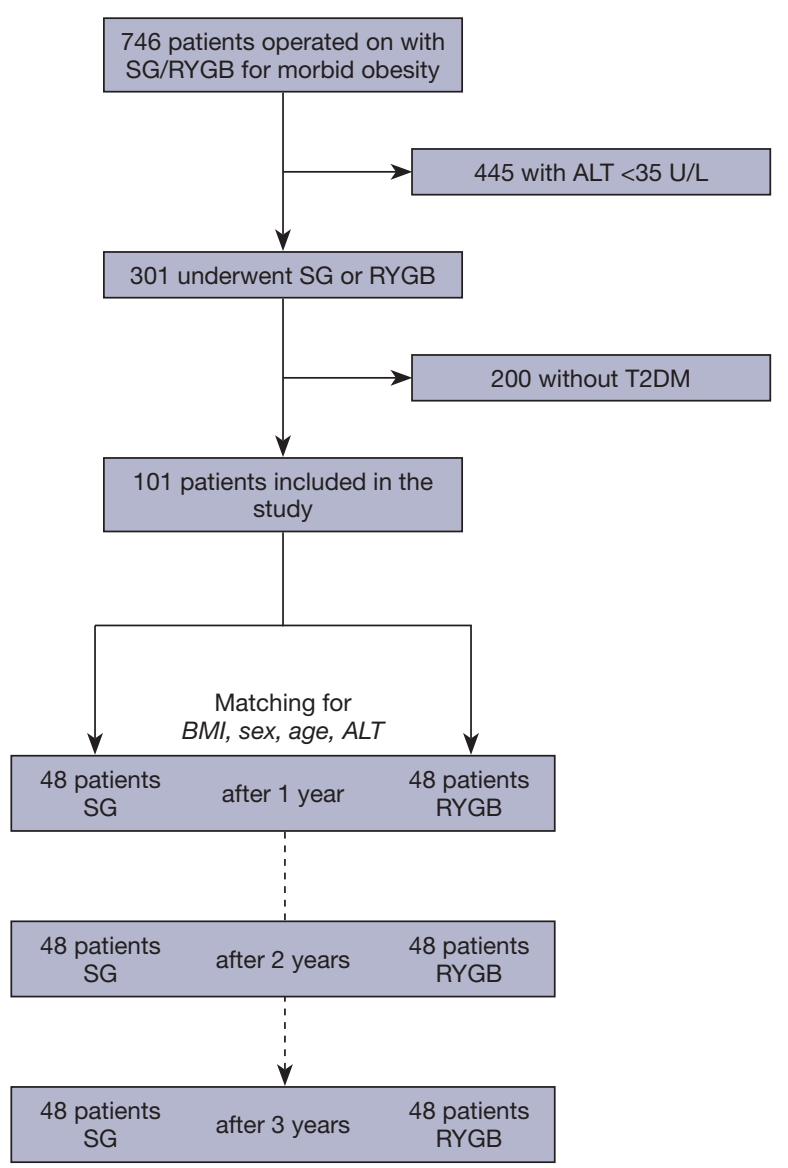

Figure 1 Flow diagram of the present study. SG, sleeve gastrectomy; RYGB, Roux-en-Y gastric bypass; ALT, alanine transaminase; T2DM, type 2 diabetes mellitus; BMI, body mass index.

a dependent variable and age ( \pm 10 years), sex, ALT/AST $( \pm 30 \mathrm{U} / \mathrm{L})$, BMI $\left( \pm 5 \mathrm{~kg} / \mathrm{m}^{2}\right)$, presence of comorbidities [Charlson comorbidity index $(\mathrm{CCI}) \pm 1$ ] as independent covariates. The cases were matched using a matching ratio of $1: 1$. One patient could only be used once for matching.

\section{Results}

\section{Patient selection, peri-operative characteristics, morbidity and mortality}

An overview of the patient selection is provided in Figure 1. The final study cohort consisted of 96 patients. Both groups (SG and RYGB) were comparable for baseline patient characteristics as intended by the matching (Table 1). The mean age was $45.4 \pm 11.5$ years in the SG and $49.7 \pm$ 10.6 years in the RYGB group $(\mathrm{P}=0.123)$. Pre-operative
BMI was $45.2 \pm 8.8 \mathrm{~kg} / \mathrm{m}^{2}$ in the SG and $42.0 \pm 9.9 \mathrm{~kg} / \mathrm{m}^{2}$ in the RYGB group $(\mathrm{P}=0.286)$. Co-morbidities, as shown by CCI, were also comparable with $1.8 \pm 1.2$ for SG and $2.2 \pm 1.1$ for RYGB $(\mathrm{P}=0.148)$. No death was observed during the entire follow-up. No statistically relevant difference in the short-term and long-term morbidity (classified according to the Clavien-Dindo classification) was observed between the groups during the follow-up period. No cases of gastric leak nor post-operative bleeding were observed. Overall, 2 clinically relevant (Clavien-Dindo Grade $\geq 2$ ) complications were ocurred, namely a trocar site hernia in the RYGB group and a reflux complication in the SG group. The latter could be managed conservatively.

\section{Weight loss was not dependent on surgical procedure}

There was no significant difference in excess weight loss (\%EWL) between the SG and RYGB cohorts during the whole follow-up period: at 1 year, \%EWL was $61.3 \% \pm 17.3 \%$ vs. $68.8 \% \pm 18.5 \%$, at 2 years, $72.9 \% \pm 11.4 \%$ vs. $74.2 \% \pm 17.5 \%$ and at 3 years, $72.2 \% \pm 10.8 \%$ vs. $73.5 \% \pm 14.5 \%$ for SG vs. RYGB respectively. In addition, there was no significant difference in \% TWL between the groups: at 1 year, $\%$ TWL was $-28.5 \% \pm 7.8 \%$ vs . $-31.4 \% \pm 7.4 \%$, at 2 years, $-26.8 \% \pm 7.2 \%$ vs. $-28.8 \% \pm 8.3 \%$ and at 3 years, $-25.7 \% \pm 7.4 \%$ vs. $-27.0 \% \pm 7.0 \%$ for SG vs. RYGB respectively.

\section{NAFLD resolved in all patients 2 years after $S G$}

There was no significant difference in AST or ALT levels pre-operatively between the two groups (Tables 1,2, Figure 2). However, using the NFS score, patients in the SG group had significantly higher scores than the RYGB group pre-operatively $(\mathrm{P}=0.009)$. This tendency was also observed using the HSI score: SG patients had significantly higher score than the RYGB group $(\mathrm{P}=0.001)$. SG and RYGB both significantly reduced ALT and AST levels after 1, 2 and 3 years compared to the pre-operative values. However, SG resulted in a larger reduction in ALT levels after 1, 2 and 3 years compared to RYGB (after 1 year, $\mathrm{P}=0.012$; after 2 years, $\mathrm{P}=0.035$; after 3 years, $\mathrm{P}=0.041$ ). This difference was not observed for AST. Furthermore, SG resulted in a biochemical resolution of NAFLD in $92.1 \%$ of patients after 1 year compared to $71.1 \%$ after RGYB ( $\mathrm{P}=0.036)$. After 2 and 3 years, NAFLD resolved in all patients after SG (100\%) compared to $78.3 \%$ after 2 years $(\mathrm{P}=0.437)$ and $80 \% 3$ years $(\mathrm{P}=0.474)$ following 
Table 1 Baseline characteristics of the 96 matched patients included in our study

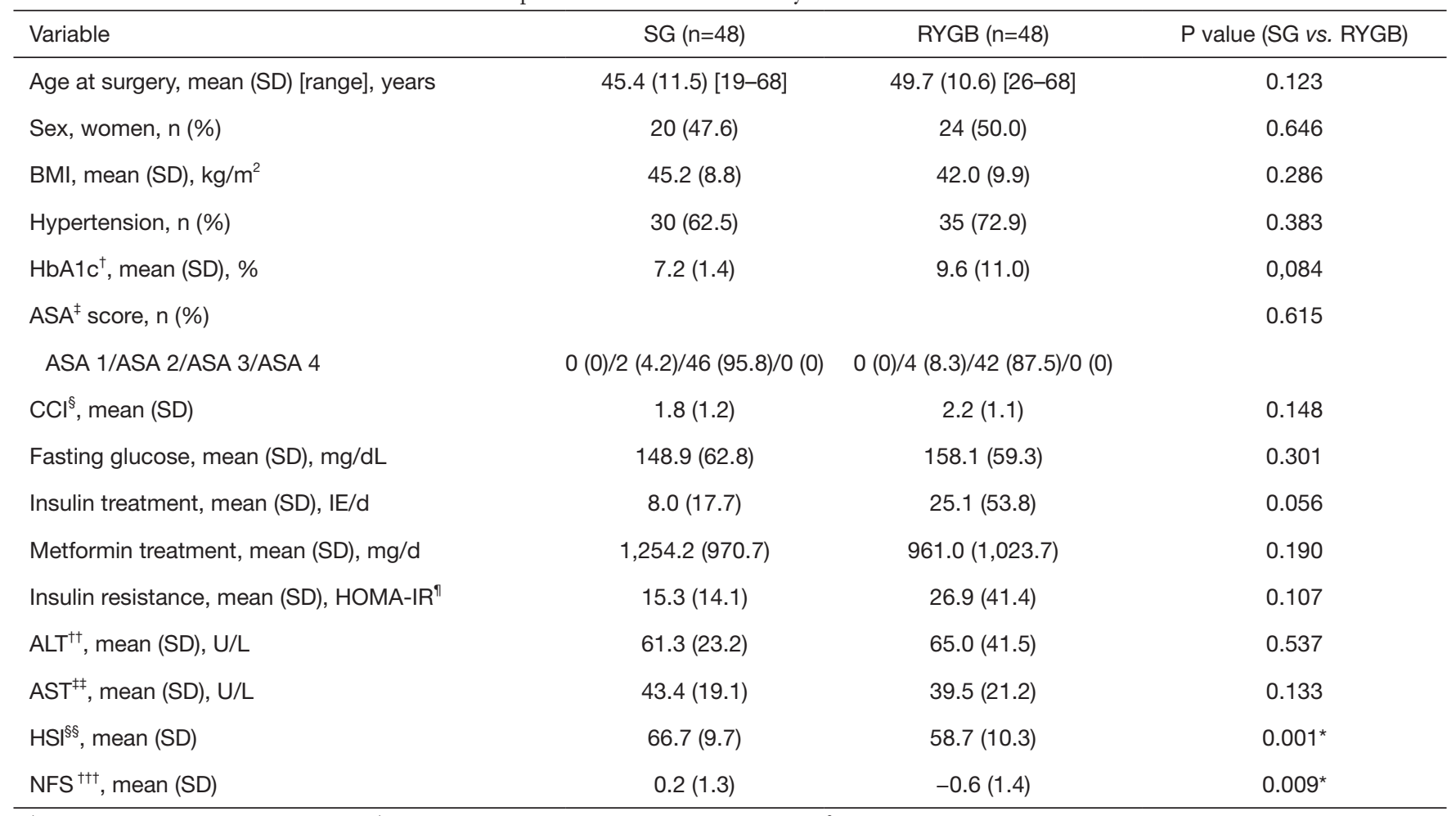

${ }^{\dagger}, \mathrm{HbA} 1 \mathrm{c}$ : glycosylated hemoglobin; ${ }^{\ddagger}, \mathrm{ASA}$ : American Society of Anesthesiologists; ${ }^{\S}$, the CCI predicts the one-year mortality for a patient who may have a range of comorbid conditions, such as heart disease, AIDS, or cancer (a total of 22 conditions). Each condition is assigned a score of 1 , 2, 3, or 6, depending on the risk of dying associated with each one. Scores are summed to provide a total score to predict mortality; ", HOMA-IR: Homeostasis Model Assessment for Insulin Resistance; ${ }^{\dagger+}$, ALT: alanine transaminase; ${ }^{\ddagger \ddagger}$, AST: aspartate aminotransferase; ${ }^{\S \S}$, HSI: Hepatic Steatosis Index; ${ }^{+\dagger+}$, NFS: NAFLD Fibrosis Score; Asterisk (*) indicates $\mathrm{P}<0.05$ between SG and RYGB. SG, sleeve gastrectomy; RYGB, Roux-en-Y gastric bypass; SD, standard deviation; BMI, body mass index; $\mathrm{CCl}$, Charlson comorbidity index; AIDS, acquired immunodeficiency syndrome.

RYGB. Similarly, both procedures led to a significant reduction in the NFS over the follow-up period (Figure 2). After the first year, the difference between both groups remained significant with lower scores in the RYGB group $(\mathrm{P}=0.007)$. From the second year onwards, this difference was no longer significant with a trend to lower scores in the SG group $(\mathrm{P}=0.419)$. Finally, from the third year onwards, the NFS was significantly lower in the SG group compared to the RYGB group ( $\mathrm{P}=0.016)$. Of note, there was no longer a difference in the NFS after 3 years compared to the pre-operative value in the RYGB group $(\mathrm{P}=0.132)$. Similarly, both procedures led to a significant reduction in the HSI score from the second postoperative year (Table 2 and Figure 2D). It is interesting to note that this difference was no longer significant from the third year onwards. From the second year onwards, it was no longer possible to make any significant difference between both groups in relation to HSI $(\mathrm{P}=0.179$ and $\mathrm{P}=0.347$ respectively at 2 and 3 years). There were no significant differences in platelet counts, serum albumin level and international normalized ratio (INR) between both groups at any follow-up point.

\section{Glycemic control and insulin resistance did not vary according to bariatric surgical approacb}

The baseline values and changes in fasting glucose, glycated hemoglobin HbA1c, medication use (insulin or metformin) and insulin resistance are shown in Tables 1,2 and Figures 3,4. Both procedures (SG and RGYB) significantly reduced HbA1c after 1 year compared to pre-operative values $(\mathrm{P}<0.001$ for $\mathrm{SG}$ and $\mathrm{P}<0.001$ for RYGB). This improvement in $\mathrm{HbA} 1 \mathrm{c}$ remained significant after 2 and 3 years in both groups irrespective of which surgical procedure was employed. Furthermore, the improved glycemic control was reflected in a reduction in oral anti-hyperglycemics 
Table 2 Follow-up characteristics

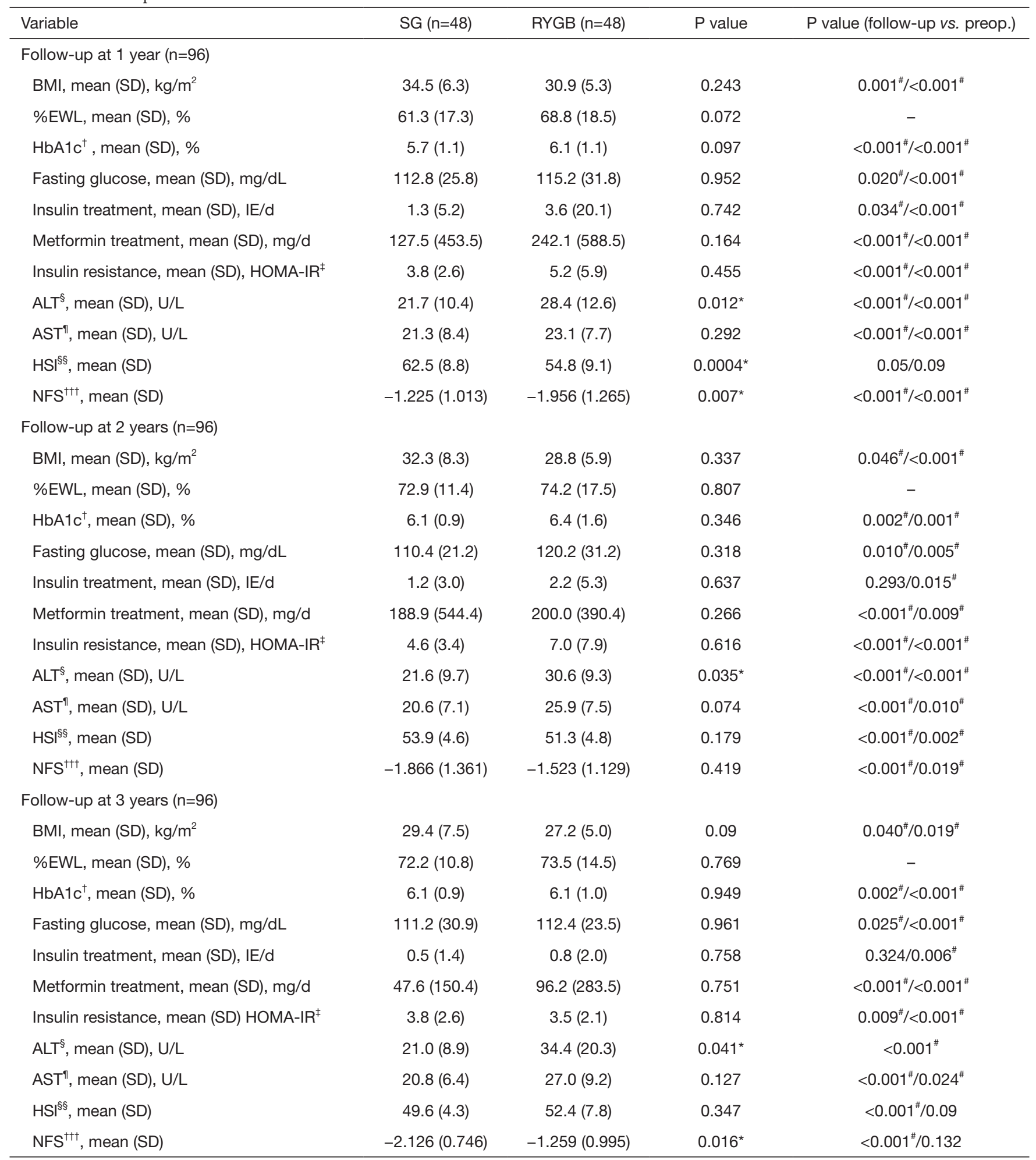

†, HbA1c: glycosylated hemoglobin; ${ }^{\ddagger}$, HOMA-IR: Homeostasis Model Assessment for Insulin Resistance; ${ }^{\S}$, ALT: alanine transaminase; ", AST: aspartate aminotransferase; ${ }^{\S \S}$, HSI: Hepatic Steatosis Index; ${ }^{\mathrm{tt \dagger}}$, NFS: NAFLD Fibrosis Score; Asterisk (*) indicates P<0.05 between $S G$ and RYGB. Hashtag $\left({ }^{*}\right)$ indicates $P<0.05$ between follow-up and preoperative value for a given procedure. SG, sleeve gastrectomy; RYGB, Roux-en-Y gastric bypass; BMI, body mass index; SD, standard deviation; EWL, excess weight loss. 

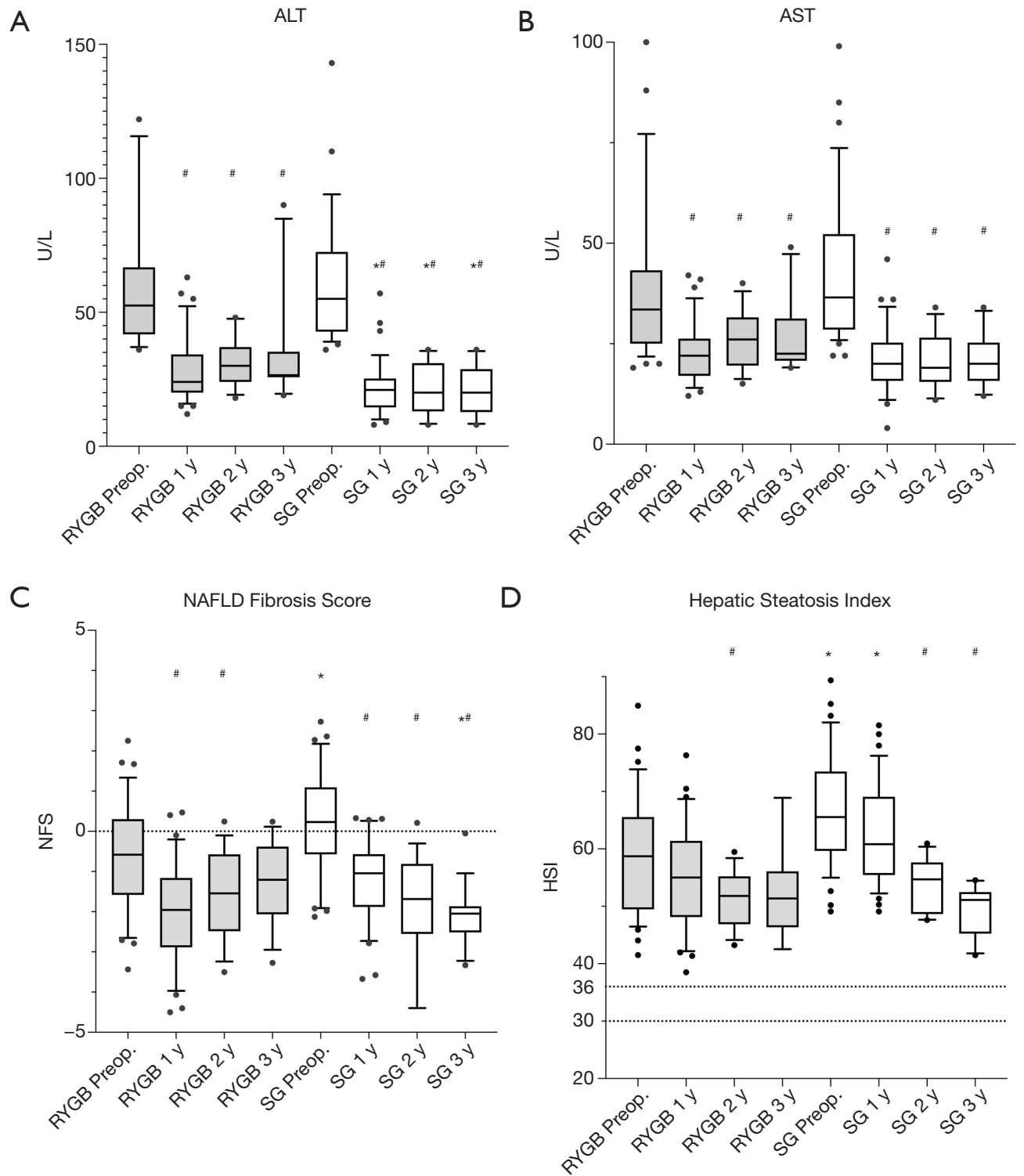

Figure 2 Changes in liver function tests. Changes in ALT (A), AST (B), NFS (C) and HSI (D) after laparoscopic SG and laparoscopic RYGB after 1,2 , and 3 years. Asterisk $\left(^{*}\right)$ indicates $\mathrm{P}<0.05$ between $\mathrm{SG}$ and RYGB. Hashtag $\left(^{*}\right)$ indicates $\mathrm{P}<0.05$ between follow-up and preoperative value for a given procedure. ALT, alanine transaminase; AST, aspartate transaminase; NFS, NAFLD Fibrosis Score; HSI, Hepatic Steatosis Index; SG, sleeve gastrectomy; RYGB, Roux-en-Y gastric bypass.

(metformin), which persisted throughout the followup period. There was no significant difference in insulin use between both study groups at any time in the followup. However, after 2 and 3 years, RYGB was associated with a significant reduction in insulin use compared to the preoperative period (Figure 4). Seventy-nine percent, 75\% and $73 \%$ of patients were off insulin or metformin after 1 , 2 and 3 years after SG, respectively, whereas $89 \%, 85 \%$ and
$83 \%$ of patients were off insulin or metformin after 1,2 and 3 years after RYGB, respectively. In addition to metformin and insulin, two patients in the SG group were treated preoperatively with Sitagliptin and another patient in the SG group received treatment with Dapagliflozin. These treatments could be discontinued after the operation.

Insulin resistance improved significantly in both groups in comparison to the pre-operative value and this 

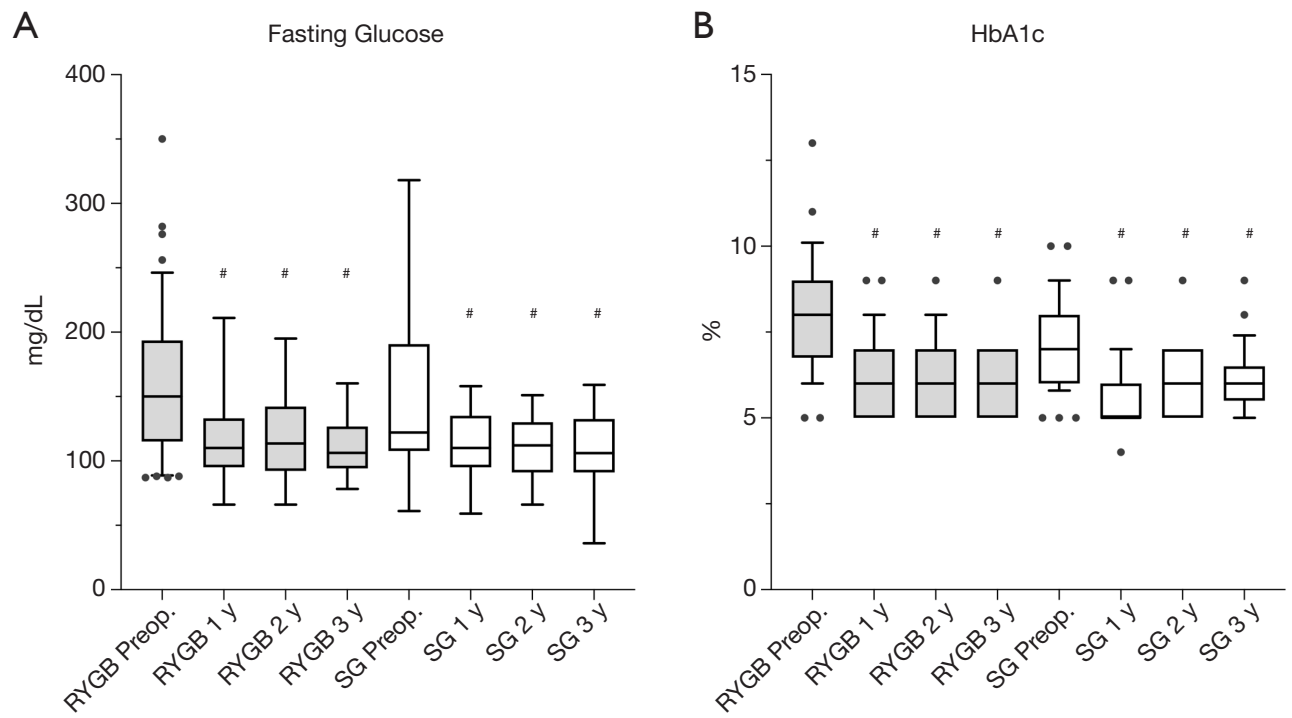

Figure 3 Changes in glycemic control and diabetes medication. Changes in fasting glucose (A) and HbA1c (B) after laparoscopic SG and laparoscopic RYGB after 1, 2, and 3 years. Hashtag $\left(^{*}\right)$ indicates $\mathrm{P}<0.05$ between follow-up and preoperative value for a given procedure. HbAlc, glycated haemoglobin; RYGB, Roux-en-Y gastric bypass; SG, sleeve gastrectomy.

improvement was maintained significantly throughout the 3 years of follow-up (Figure 4). For SG, HOMA-IR improved significantly at 1 year $(\mathrm{P}<0.001), 2$ years $(\mathrm{P}<0.001)$ and 3 years $(\mathrm{P}=0.009)$. For RYGB, this index improved significantly at 1 year $(\mathrm{P}<0.001), 2$ years $(\mathrm{P}=0.0002)$ and 3 years $(\mathrm{P}<0.001)$. According to the 2009 American Diabetes Association (ADA) consensus definition, complete T2DM remission was achieved in $43.8 \%$ of patients after 1 year, $39.2 \%$ of patients after 2 years and $40.5 \%$ of patients after 3 years. There was no significant difference between the two procedures throughout the 3 years of follow-up (after 1 year $\mathrm{P}=0.149$; after 2 years $\mathrm{P}=0.153$; after 3 years $\mathrm{P}=0.100$ ).

\section{Discussion}

Patients with NAFLD and DM are at increased risk of progressing from steato-hepatitis to irreversible fibrosis and ultimately hepatocellular cancer. Whilst weight loss surgery may be halt this progression, there is currently a lack of evidence regarding which specific bariatric surgical approach may be best suited to particular patient populations in terms of ameliorating metabolic dysfunction.

Liver biopsy is the gold standard for the diagnosis and follow-up of obesity related liver disease (e.g., NASH). To the best of our knowledge only 3 studies have used liver biopsies to follow-up the effects of SG and RYGB on NAFLD $(14,17,26)$. These studies, together with the recent meta-analysis by Panunzi et al. (27), were unable to demonstrate superiorty of any particular surgical technique on retarding the progression of NAFLD. Given that histological confirmation of NAFLD is not without risks, including severe haemorrhage, there has been a move towards non-invasive methods for assessment of liver steatosis and fibrosis (e.g., ALT/AST level, NAFLD Fibrosis Score, Bard Score, elastography, MRI) $(1,28)$. Non-invasive imaging, for example MRI, has become the gold standard stage in the follow-up of NAFLD. Moreover, the majority of biopsy techniques (e.g., wedge resection) also sample subcapsular hepatic parenchyma and are prone to histological misinterpretation due to the presence of normal subcapsular fibrosis. Therefore, the combination of clinical scores and plasma biomarkers for the detection of NAFLD, NASH or advanced fibrosis may provide a reliable alternative to minimize the need for liver biopsies (29).

It is important to point out the evidence-base to date is conflicting (30,31). Billeter et al. reported that SG was superior to RYGB in terms of its reduction in markers of liver function in patients with combined NAFLD and T2DM, whereas both procedures had comparable effects on improving glycemic control in patients with T2DM (15). The beneficial effects of SG in patients with histologically proven NASH were confirmed in a randomized trial, at least 
A

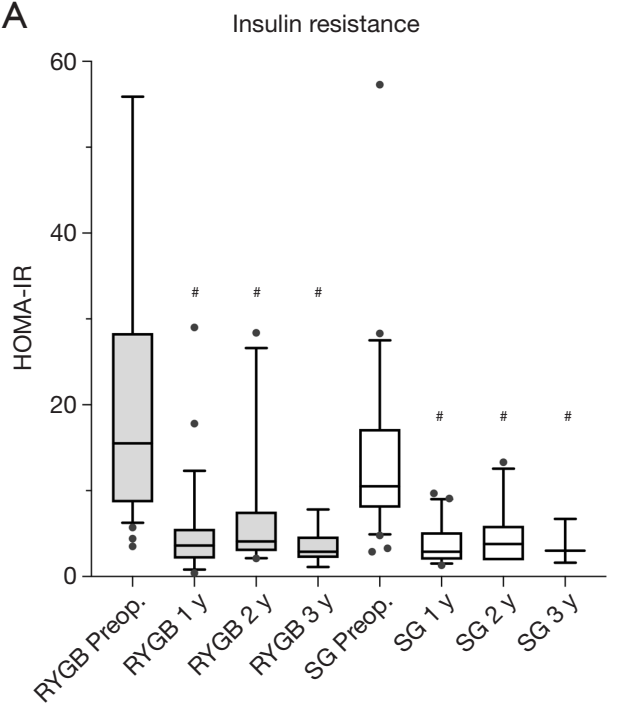

C

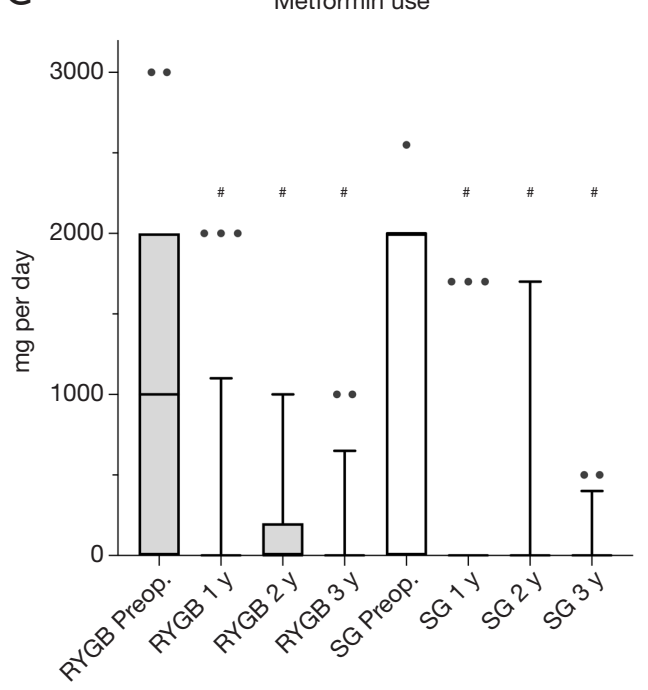

B

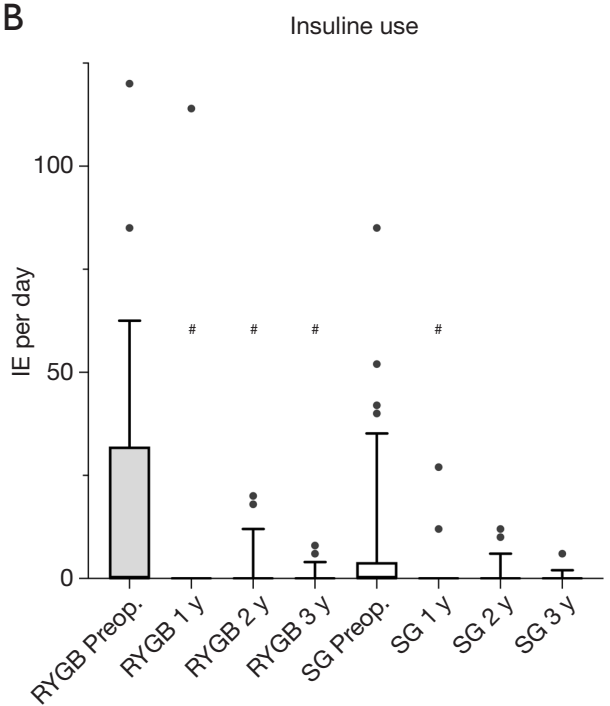

Figure 4 Changes in insulin resistance, glycemic control and diabetes medication. Changes in HOMA-IR (A), insulin use (B) and metformin use (C) after laparoscopic SG and laparoscopic RYGB after 1, 2, and 3 years. Hashtag $(")$ indicates $\mathrm{P}<0.05$ between follow-up and preoperative value for a given procedure. RYGB, Roux-en-Y gastric bypass; SG, sleeve gastrectomy; HOMA-IR, Homeostasis Model Assessment for Insulin Resistance.

in terms of normalization of liver enzymes (16). A recent meta-analysis showed a strong but non-significant benefit of SG over RYGB in terms of reduction in transaminases (30). Moreover, 12 months after SG, $100 \%$ of patients experienced resolution or improvement in NAFLD/ NASH as well as fibrosis, with just $16 \%$ of patients showing steatosis at that time (in comparison to preoperative where all patients showed steatosis) (32). In contrast, RYGB may actually lead to decompensation of liver cirrhosis in up to $4 \%$ of cases (33). Liver failure after bariatric surgery may be due to the increased degree of malabsorption (i.e., malnutrition) attributed to procedures like RYGB, when compared to SG (34). Another randomized trial (17) suggested a negative impact of RYGB on markers of hepatic synthetic function (INR, albumin). The exact mechanisms by which RYGB may lead to decompensation is not fully elucidated; 
however, malabsorption or bacterial overgrowth might play key pathophysiological roles $(35,36)$.

In order to provide more robust evidence of NAFLD improvement after metabolic surgery we used the NFS and HSI in combination to ALT/AST levels (22). Preoperatively, the HSI score (evaluating liver steatosis) was significantly worse in the SG group, but the improvement was more pronounced when compared to that in the RYGB cohort after 2 and 3 years. This could also be observed when investigating the NFS score (particularly suited to evaluate liver fibrosis). This suggests that patients in the SG group were at a higher risk of steatosis and fibrosis pre-operatively, which seemed to be more amenable to treatment with SG than RYGB. These findings are highly clinically relevant given that liver fibrosis itself is the strongest predictor for advanced liver disease (37). However, it should be noted that even after post-operative improvement in the HSI scores for both procedures, they remain above the threshold value for steatosis. Thus, these results should be corroborated using investigations such as MRI or sonography.

Although weight loss by itself can lead to an improvement or resolution in NAFLD (12), impacting several critical signalling pathways and metabolic pathways (38), mechanisms independent of weight loss have also been postulated. Post-operative changes in bile acid enterohepatic circulation (greater apical sodium bile salt transporterstained area, increased villi length, total surface area), as well as intestinal hormone (Ghrelin-O-acyltransferase) and growth factor secretion $(38,39)$ have been shown to play a significant role. Recently, an additional microbiotamediated mechanism was proposed to have a procedurespecific impact on NAFLD, through post-operative selection of specific microbial species, its impact on bile acid ratio and signalling pathway activation $(38,40,41)$.

In addition to efficacy, safety also plays a fundamental role when deciding which surgical procedure to employ. While bypass-procedures may be associated with a higher risk of liver decompensation in patients with severe liver damage, liver failure was not observed following SG (42). Indeed, several studies instead indicate that mechanisms independent of NAFLD (e.g., perturbation in gut permeability or microbiota changes) may negatively affect liver function following RYGB (43). Therefore, it is at least conceivable that SG may be safer for patients with extensive pre-operative liver disease.

With regards to T2DM, investigations have shown that the effect of bariatric procedures on the correction of diabetes is in part directly related to weight loss (44). Lean et al. (44) were able to show that an integrated structured weight-management programme sustained remissions at 24 months for more than a third of people with type 2 diabetes. Although weight loss is considered the dominant cause, actual results support the involvement of weightindependent mechanisms.

Our results are consistent with the published literature, confirming that SG and RYGB achieve comparable improvements of glycemic control $(15,45)$. Although recent meta-analyses report short-term results (at 1 year) in favour of RYGB (46), both procedures had similar effects on insulin resistance, as determined by the HOMA-IR, over the 3 years of follow-up (47). In a long-term study in an Asian study population, authors showed that SG was associated with a more marked improvement in T2DM than RYGB (48). This result was also confirmed in a recent network meta-analysis, which compared single anastomosis gastric bypass, RYGB and SG (49). While it is undisputable that metabolic surgery, in general, has strong effects on T2DM-associated complications (9,15-17), there are currently no prospective long-term studies which have compared the effects of SG vs. RYGB on T2DM-related complications, nor series showing any significant difference in terms of cardiovascular or microvascular endpoints or mortality. Thus, the clinical relevance of the greater improvements in glycemic control attributed to RYGB remains to be determined (50). Moreover, the differences found when comparing SG to RYGB only focussed on patients with T2DM as the only comorbidity (6). Bearing in mind that SG may be safer in patients with advanced liver disease, our series provides evidence that SG may be preferable to RYGB for T2DM control in obese patients with both T2DM and NAFLD.

The main limitation of the present study is its retrospective design. This may have resulted in an element of selection bias given that the surgeons and patients chose the type of operation and it was not selected randomly. In order to compensate for this bias, both cohorts were matched. However, it is important to recognise that the matching process used in our series is no replacement for a prospective randomized study design. A consequence of matching was that pre-operative liver enzyme levels (AST), HSI score and NFS were worse in the SG group compared to RYGB group. Nevertheless, SG patients achieved significantly better results than RYGB patients for these variables after 2 and 3 years. The claimed greater effect on liver function tests and Steatosis/Fibrosis score is likely to be an effect of tendency to the mean-the baseline values being modestly greater in the SG group and the final 
values similar. The importance of our clinical observations lies in the considerable and clinical important changes brought about by both surgical procedures. Thus, it is not unreasonable to point out the slightly greater fall in the SG group as this runs counter to what many authorities working in this field might expect.

Sampling errors can significantly affect the precision and interpretation of results. Our sample size is small and may lead to bias. However, we performed a post-hoc power calculation to verify that the number of patients included in our series allowed us to observe significant effects with sufficient power. Given that the study design was based on two independent study groups (SG vs. RYGB), and taking account of the anticipated means of ALT and AST (25) (type I/II error rate: alpha $=0.05$ and beta $=0.2$, power of $80 \%$ ) a minimum sample size (Rosner's method) of $n=13$ patients for each group (total study group $=26$ ) was necessary.

Lack of histological follow-up (discussed above) is another limitation of our study. The current consensus in the literature for relatively low risk pathology (such as NAFLD) is that such a histopathologic follow-up is not justified (34) and most prospective series do not use liver biopsies for monitoring NAFLD.

\section{Conclusions}

In conclusion, despite its inherent limitations, our study suggests that SG may offer advantages in the treatment of obese patients with combined NAFLD and T2DM when compared with RYGB. SG achieved a more pronounced reduction in liver enzymes and markers of steatosis and fibrosis while there was no difference between SG and RYGB in terms of glycemic control. Given that both procedures had similar effects regarding weight loss and complications rates, SG may be more suited to obese patients with both NAFLD and T2DM, especially those with severe metabolic dysfunction and advanced liver disease. Whilst this needs to be confirmed in prospective studies, taking patient co-morbidities and procedure-specific metabolic effects of bariatric surgery into consideration may help optimise surgery selection criteria to improve patient outcomes and safety.

\section{Acknowledgments}

The authors would like to thank PD Dr. med. Ewan A. Langan for his advice on qualitative study design and English language proof-checking.

Funding: None.

\section{Footnote}

Reporting Checklist: The authors have completed the STROBE reporting checklist. Available at https://hbsn. amegroups.com/article/view/10.21037/hbsn-21-71/rc

Data Sharing Statement: Available at https://hbsn. amegroups.com/article/view/10.21037/hbsn-21-71/dss

Conflicts of Interest: All authors have completed the ICMJE uniform disclosure form (available at https://hbsn. amegroups.com/article/view/10.21037/hbsn-21-71/coif). The authors have no conflicts of interest to declare.

Ethical Statement: The authors are accountable for all aspects of the work in ensuring that questions related to the accuracy or integrity of any part of the work are appropriately investigated and resolved. The study was conducted in accordance with the Declaration of Helsinki (as revised in 2013). The study was approved by institutional review board (University of Heidelberg) and individual consent for this retrospective analysis was waived.

Open Access Statement: This is an Open Access article distributed in accordance with the Creative Commons Attribution-NonCommercial-NoDerivs 4.0 International License (CC BY-NC-ND 4.0), which permits the noncommercial replication and distribution of the article with the strict proviso that no changes or edits are made and the original work is properly cited (including links to both the formal publication through the relevant DOI and the license). See: https://creativecommons.org/licenses/by-nc-nd/4.0/.

\section{References}

1. Younossi ZM, Koenig AB, Abdelatif D, et al. Global epidemiology of nonalcoholic fatty liver disease-Metaanalytic assessment of prevalence, incidence, and outcomes. Hepatology 2016;64:73-84.

2. Wang YC, McPherson K, Marsh T, et al. Health and economic burden of the projected obesity trends in the USA and the UK. Lancet 2011;378:815-25.

3. Berzigotti A, Garcia-Tsao G, Bosch J, et al. Obesity is an independent risk factor for clinical decompensation in patients with cirrhosis. Hepatology 2011;54:555-61.

4. Adams TD, Davidson LE, Litwin SE, et al. Weight and Metabolic Outcomes 12 Years after Gastric Bypass. N Engl J Med 2017;377:1143-55. 
5. Courcoulas AP, King WC, Belle SH, et al. SevenYear Weight Trajectories and Health Outcomes in the Longitudinal Assessment of Bariatric Surgery (LABS) Study. JAMA Surg 2018;153:427-34.

6. Schauer PR, Bhatt DL, Kirwan JP, et al. Bariatric Surgery versus Intensive Medical Therapy for Diabetes - 5-Year Outcomes. N Engl J Med 2017;376:641-51.

7. Madsbad S, Dirksen C, Holst JJ. Mechanisms of changes in glucose metabolism and bodyweight after bariatric surgery. Lancet Diabetes Endocrinol 2014;2:152-64.

8. Müller-Stich BP, Senft JD, Warschkow R, et al. Surgical versus medical treatment of type 2 diabetes mellitus in nonseverely obese patients: a systematic review and metaanalysis. Ann Surg 2015;261:421-9.

9. Billeter AT, Scheurlen KM, Probst P, et al. Metaanalysis of metabolic surgery versus medical treatment for microvascular complications in patients with type 2 diabetes mellitus. Br J Surg 2018;105:168-81.

10. Chalasani N, Younossi Z, Lavine JE, et al. The diagnosis and management of nonalcoholic fatty liver disease: Practice guidance from the American Association for the Study of Liver Diseases. Hepatology 2018;67:328-57.

11. Lee Y, Doumouras AG, Yu J, et al. Complete Resolution of Nonalcoholic Fatty Liver Disease After Bariatric Surgery: A Systematic Review and Meta-analysis. Clin Gastroenterol Hepatol 2019;17:1040-60.e11.

12. Al-Mrabeh A, Zhyzhneuskaya SV, Peters C, et al. Hepatic Lipoprotein Export and Remission of Human Type 2 Diabetes after Weight Loss. Cell Metab 2020;31:233-49.e4.

13. Lassailly G, Caiazzo R, Ntandja-Wandji LC, et al. Bariatric Surgery Provides Long-term Resolution of Nonalcoholic Steatohepatitis and Regression of Fibrosis. Gastroenterology 2020;159:1290-301.e5.

14. Froylich D, Corcelles R, Daigle C, et al. Effect of Roux-en-Y gastric bypass and sleeve gastrectomy on nonalcoholic fatty liver disease: a comparative study. Surg Obes Relat Dis 2016;12:127-31.

15. Billeter AT, Senft J, Gotthardt D, et al. Combined Nonalcoholic Fatty Liver Disease and Type 2 Diabetes Mellitus: Sleeve Gastrectomy or Gastric Bypass?-a Controlled Matched Pair Study of 34 Patients. Obes Surg 2016;26:1867-74.

16. Kalinowski P, Paluszkiewicz R, Ziarkiewicz-Wróblewska B, et al. Liver Function in Patients With Nonalcoholic Fatty Liver Disease Randomized to Roux-en-Y Gastric Bypass Versus Sleeve Gastrectomy: A Secondary
Analysis of a Randomized Clinical Trial. Ann Surg 2017;266:738-45.

17. von Schönfels W, Beckmann JH, Ahrens M, et al. Histologic improvement of NAFLD in patients with obesity after bariatric surgery based on standardized NAS (NAFLD activity score). Surg Obes Relat Dis 2018;14:1607-16.

18. Handelsman Y, Bloomgarden ZT, Grunberger G, et al. American association of clinical endocrinologists and American college of endocrinology - clinical practice guidelines for developing a diabetes mellitus comprehensive care plan - 2015. Endocr Pract 2015;21:S1-S87.

19. AWMF, DGAV-CAADIP. S3-Leitlinie: Chirurgie der Adipositas und metabolischer Erkrankungen. Available online: https://www.awmf.org/uploads/tx_szleitlinien/0880011_S3_Chirurgie-Adipositas-metabolischeErkrankugen_2018-02.pdf

20. Kleiner DE, Berk PD, Hsu JY, et al. Hepatic pathology among patients without known liver disease undergoing bariatric surgery: observations and a perspective from the longitudinal assessment of bariatric surgery (LABS) study. Semin Liver Dis 2014;34:98-107.

21. Prati D, Taioli E, Zanella A, et al. Updated definitions of healthy ranges for serum alanine aminotransferase levels. Ann Intern Med 2002;137:1-10.

22. Yeo SC, Ong WM, Cheng KSA, et al. Weight Loss After Bariatric Surgery Predicts an Improvement in the Nonalcoholic Fatty Liver Disease (NAFLD) Fibrosis Score. Obes Surg 2019;29:1295-300.

23. Angulo P, Hui JM, Marchesini G, et al. The NAFLD fibrosis score: a noninvasive system that identifies liver fibrosis in patients with NAFLD. Hepatology 2007;45:846-54.

24. Matthews DR, Hosker JP, Rudenski AS, et al. Homeostasis model assessment: insulin resistance and beta-cell function from fasting plasma glucose and insulin concentrations in man. Diabetologia 1985;28:412-9.

25. Buse JB, Caprio S, Cefalu WT, et al. How do we define cure of diabetes? Diabetes Care 2009;32:2133-5.

26. Praveen Raj P, Gomes RM, Kumar S, et al. The effect of surgically induced weight loss on nonalcoholic fatty liver disease in morbidly obese Indians: "NASHOST" prospective observational trial. Surg Obes Relat Dis 2015;11:1315-22.

27. Panunzi S, Maltese S, Verrastro O, et al. Pioglitazone and bariatric surgery are the most effective treatments for non-alcoholic steatohepatitis: A hierarchical network 
meta-analysis. Diabetes Obes Metab 2021;23:980-90.

28. Kinner S, Reeder SB, Yokoo T. Quantitative Imaging Biomarkers of NAFLD. Dig Dis Sci 2016;61:1337-47.

29. Bril F, McPhaul MJ, Caulfield MP, et al. Performance of Plasma Biomarkers and Diagnostic Panels for Nonalcoholic Steatohepatitis and Advanced Fibrosis in Patients With Type 2 Diabetes. Diabetes Care 2020;43:290-7.

30. Fakhry TK, Mhaskar R, Schwitalla T, et al. Bariatric surgery improves nonalcoholic fatty liver disease: a contemporary systematic review and meta-analysis. Surg Obes Relat Dis 2019;15:502-11.

31. Perysinakis I, Pappis HC, Margaris E. Current Controversies in Metabolic Surgery for Nonalcoholic Fatty Liver Disease. Obes Surg 2019;29:1058-67.

32. Esquivel CM, Garcia M, Armando L, et al. Laparoscopic Sleeve Gastrectomy Resolves NAFLD: Another Formal Indication for Bariatric Surgery? Obes Surg 2018;28:4022-33.

33. Jan A, Narwaria M, Mahawar KK. A Systematic Review of Bariatric Surgery in Patients with Liver Cirrhosis. Obes Surg 2015;25:1518-26.

34. Mahawar KK, Parmar C, Graham Y, et al. Monitoring of Liver Function Tests after Roux-en-Y Gastric Bypass: An Examination of Evidence Base. Obes Surg 2016;26:2516-22.

35. Bal BS, Finelli FC, Shope TR, et al. Nutritional deficiencies after bariatric surgery. Nat Rev Endocrinol 2012;8:544-56.

36. Dolan RD, Baker J, Harer K, et al. Small Intestinal Bacterial Overgrowth: Clinical Presentation in Patients with Roux-en-Y Gastric Bypass. Obes Surg 2021;31:564-9.

37. Hagström H, Nasr P, Ekstedt M, et al. Fibrosis stage but not NASH predicts mortality and time to development of severe liver disease in biopsy-proven NAFLD. J Hepatol 2017;67:1265-73.

38. Kohli R, Myronovych A, Tan BK, et al. Bile Acid Signaling: Mechanism for Bariatric Surgery, Cure for NASH? Dig Dis 2015;33:440-6.

39. Habegger KM, Al-Massadi O, Heppner KM, et al. Duodenal nutrient exclusion improves metabolic syndrome and stimulates villus hyperplasia. Gut 2014;63:1238-46.

40. Sookoian S, Salatino A, Castaño GO, et al. Intrahepatic bacterial metataxonomic signature in non-alcoholic fatty liver disease. Gut 2020;69:1483-91.

41. Xu G, Song M. Recent advances in the mechanisms underlying the beneficial effects of bariatric and metabolic surgery. Surg Obes Relat Dis 2021;17:231-8.

42. Eilenberg M, Langer FB, Beer A, et al. Significant Liver-Related Morbidity After Bariatric Surgery and Its Reversal-a Case Series. Obes Surg 2018;28:812-9.

43. Ledoux S, Sami O, Calabrese D, et al. Gastric bypass specifically impairs liver parameters as compared with sleeve gastrectomy, independently of evolution of metabolic disorders. Surg Obes Relat Dis 2019;15:220-6.

44. Lean MEJ, Leslie WS, Barnes AC, et al. Durability of a primary care-led weight-management intervention for remission of type 2 diabetes: 2 -year results of the DiRECT open-label, cluster-randomised trial. Lancet Diabetes Endocrinol 2019;7:344-55.

45. Sharples AJ, Mahawar K. Systematic Review and MetaAnalysis of Randomised Controlled Trials Comparing Long-Term Outcomes of Roux-En-Y Gastric Bypass and Sleeve Gastrectomy. Obes Surg 2020;30:664-72.

46. Madadi F, Jawad R, Mousati I, et al. Remission of Type 2 Diabetes and Sleeve Gastrectomy in Morbid Obesity: a Comparative Systematic Review and Meta-analysis. Obes Surg 2019;29:4066-76.

47. Sheka AC, Adeyi O, Thompson J, et al. Nonalcoholic Steatohepatitis: A Review. JAMA 2020;323:1175-83.

48. Huang YM, Lin YK, Lee WJ, et al. Long-term outcomes of metabolic surgery in overweight and obese patients with type 2 diabetes in Asia. Diabetes Obes Metab 2021;23:742-53.

49. Cresci B, Cosentino C, Monami M, et al. Metabolic surgery for the treatment of type 2 diabetes: A network meta-analysis of randomized controlled trials. Diabetes Obes Metab 2020;22:1378-87.

50. Lee Y, Doumouras AG, Yu J, et al. Laparoscopic Sleeve Gastrectomy Versus Laparoscopic Roux-en-Y Gastric Bypass: A Systematic Review and Meta-analysis of Weight Loss, Comorbidities, and Biochemical Outcomes From Randomized Controlled Trials. Ann Surg 2021;273:66-74.

Cite this article as: Billmann F, El Shishtawi S, Bruckner T, ElSheikh M, Müller-Stich BP, Billeter A. Combined nonalcoholic fatty liver disease and type 2 diabetes in severely obese patients-medium term effects of sleeve gastrectomy versus Roux-en-Y-gastric bypass on disease markers. HepatoBiliary Surg Nutr 2022;11(6):795-807. doi: 10.21037/hbsn-21-71 